\title{
Primary lymphosarcoma of lung associated with hypertrophic pulmonary osteoarthropathy
}

\author{
G F A BENFIELD
}

From Ham Green Hospital, Ham Green, Bristol BS20 OHW, UK

\section{Case report}

A 51-year-old woman presented in August 1971 with a two year history of slight left chest pain, mild exertional dyspnoea, and a productive cough for six weeks. There had been no constitutional disturbance. Chest radiography showed an almost totally opaque left hemithorax and an air bronchogram with homogeneous consolidation of the left upper lobe and most of the left lower lobe. The sputum was mucoid, yielded normal respiratory flora on culture, and contained no malignant cells or acid fast bacilli. Bronchoscopy showed no abnormality. Vital capacity (VC) was $2 \cdot 21$ (predicted 3.71 ) and the FEV 1.95 1. The haemoglobin concentration was $12.8 \mathrm{~g} / \mathrm{dl}$, the white cell count $6.4 \times 10^{9} / 1$ with a normal differential count, and the sedimentation rate $21 \mathrm{~mm}$ in one hour.

The patient received physiotherapy and antibiotics and remained well and at work, although by November 1974 she had become noticeably more short of breath on exertion and had developed an irritating, but largely unproductive, cough. At that time the VC had fallen to 1.751 and the FEV to $1 \cdot 31$. Chest radiography showed increasing opacification of the left lower lobe. Areas of infiltration had also developed in the mid-zone of the right lung (fig 1). The patient's weight had so far remained constant, but she lost $10 \mathrm{~kg}$ during the next eight months and towards the end of this period she developed painful swelling of the ankles, wrists, and fingers. Finger clubbing was noted and radiographs of the ankles and wrists showed changes typical of hypertrophic pulmonary osteoarthropathy with formation of periosteal new bone around the ends of the shafts of the long bones (fig 2). The latex slide test result was negative. A lung scan showed no perfusion and no ventilation of the left lung. There was also slightly diminished perfusion and ventilation at the base of the right lung. Sputum culture yielded normal flora, the white cell count had risen to $18 \cdot 1 \times 10^{9} / 1$ with a normal differential, and the sedimentation rate had risen to $70 \mathrm{~mm}$ in one hour.

Left pleuropneumonectomy with removal of

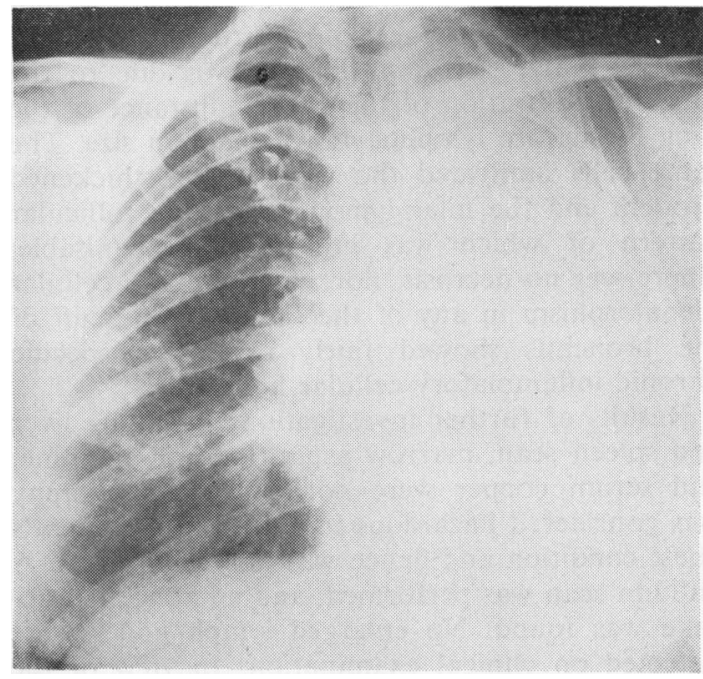

Fig 1 Chest radiograph on presentation showing completely opaque left hemithorax and right lung infiltration.

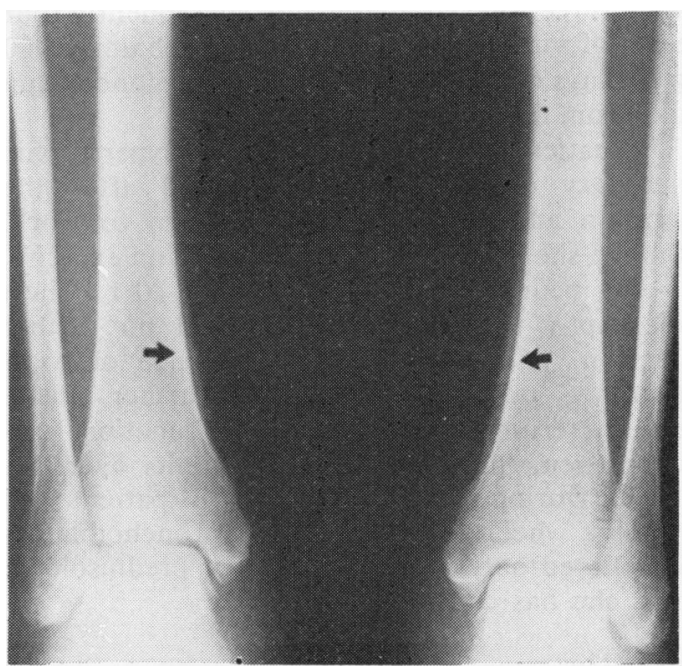

Fig 2 Typical changes of hypertrophic pulmonary osteoarthropathy in tibiae. 
the hilar lymph nodes was carried out in July 1976. The whole left lung was consolidated, and the cut surface was greyish in colour and quite uniform apart from the presence of one or two small irregular cystic cavities near the pleural surface. The pleura was slightly thickened, and there were some slightly enlarged lymph nodes at the hilum. The upper and lower lobe bronchial walls were thickened, but the main bronchus appeared normal. The thickening of the lobar bronchi was associated with some narrowing, but there was no evidence of obstructing tumour. Histologically, the consolidation was due to the uniform infiltration of the entire substance of the lung by mature lymphocytes of medium size. The same cells infiltrated the walls of the thickened bronchi and the hilar lymph nodes, the follicular pattern of which was no longer recognisable. There was no necrosis, nor was there any cellular pleomorphism in any of the sections. The cuff of the bronchus showed fairly severe nonspecific chronic inflammatory cellular infiltration.

Results of further investigations including liver and spleen scan, marrow aspiration and trephine, and serum copper were normal. Lymphography was considered hazardous in view of the patient's chest condition and hence was not undertaken. A gallium scan was performed, and no abnormal uptake was found. No enlarged lymph nodes were detected on clinical examination. In view of the patient's poor lung function and the lack of evidence of mediastinal disease radiotherapy was withheld. Chemotherapy lasting ten months was started in late August 1976 and consisted of cycles lasting three weeks initially, and subsequently four weeks, of vincristine $2 \mathrm{mg}$, cyclophosphamide 900 $\mathrm{mg}$ on days one and eight, and prednisolone $40 \mathrm{mg}$ a day from days one to eight.

The patient's finger clubbing and hypertrophic pulmonary osteoarthropathy resolved after the operation and she was able to return to work, although she was more short of breath on exercise, her VC being 1.51 and her FEV 1.11 . Chest radiography two years later showed no further extension, and there was no evidence of local recurrence or of dissemination further afield. Regular serum copper and liver function estimations remained within normal limits over this period. During chemotherapy the patient did develop a wheeze uninfluenced by bronchodilators and relieved only by $10 \mathrm{mg}$ daily of prednisolone, which she has continued to take.

\section{Discussion}

Primary lymphosarcoma of the lung was first reported in 1926 and subsequent recorded instances of this rare disease number barely one hundred, although several reviews have appeared at intervals (Havard et al, 1962; Papaioannou and Watson, 1965; Jenkins and Salm, 1971; Rees, 1973).

The clinical and radiological features of the disease do not aid diagnosis, which is almost invariably made on histological examination (Papaioannou and Watson, 1965). The rare occurrence of uncommon features such as amyloid (Jenkins and Salm, 1971) and abnormal globulins (Hurt and Kennedy, 1974) emphasises the diagnostic difficulties. This additional case shows the hitherto unrecorded clinical features of clubbing and hypertrophic pulmonary osteoarthropathy. Until now this feature has been conspicuously absent (Jenkins and Salm, 1971; Rees, 1973).

The formal cytotoxic drug regimen was given with the object of treating possible dissemination, either to the contralateral lung or beyond. Previously chemotherapy had been confined to cases with local or widespread recurrences (Rees, 1973; Hurt and Kennedy, 1974) and one case of extensive pulmonary disease where it was the sole treatment (Rees, 1973). A five-year survival rate of $44 \%$ after resection (Papaioannou and Watson, 1965), however, means that it will be some time before practical conclusions can be reached regarding prophylactic chemotherapy.

I am most grateful to Dr R A Craig for permission to publish details of this patient.

\section{References}

Jenkins, B A G, and Salm, R (1971). Primary lymphosarcoma of the lung. British Journal of Diseases of the Chest, 65, 225-237.

Havard, C W H, Nichols, J B, and Stansfeld, A G (1962). Primary lymphosarcoma of the lung. Thorax, 17, 190-200.

Hurt, R L, and Kennedy, W P U (1974). Primary lymphosarcoma of the lung. Thorax, 29, 258-261. N

Papaioannou, A N, and Watson, W L (1965). Primary lymphoma of the lung. An appraisal of its natural $\tilde{O}$ history and a comparison with other localised $\underset{\omega}{N}$ lymphomas. Journal of Thoracic and Cardiovascular

Surgery, 49, 373-387.
Rees, G M (1973). Primary lymphosarcoma of the $\frac{0}{\mathbb{C}}$ lung. Thorax, 28, 429-432.

Requests for reprints to: Dr G F A Benfield, Plymouth General Hospital, North Friary House, Greenbank Terrace, Plymouth PL4 8QQ. 